\title{
Synchrotron X-ray In Situ Tomography of Thermally Induced Phase Separation of Polylactic Acid in 1,4-Dioxane Solution
}

\author{
Swann Gay, ${ }^{\dagger \neq}$ Brice Calvignac, ${ }^{\dagger}$ Landry Ouanssi Kamtcheu, ${ }^{\ddagger}$ Thomas Beuvier, ${ }^{\ddagger}$ Elodie Boller, ${ }^{\S}$

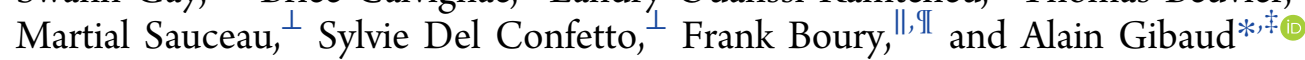 \\ †Micro \& Nanomédecines Translationelles-MINT, Université Angers, UBL Université Bretagne Loire, INSERM U1066, CNRS \\ UMR 6021, Angers F-49933 France \\ ${ }^{\ddagger}$ LUNAM University, CNRS UMR 6283-Institut des Matériaux et des Molécules du Mans, 72085 Le Mans Cedex 09, France \\ ${ }^{\S}$ ESRF, 71 avenue des Martyrs, 38000 Grenoble, France \\ "Laboratory INSERM U1232, Center for Cancer and Immunology Research, Team 17, University of Angers, Angers 49933, France \\ ${ }^{\perp}$ Université de Toulouse, Ecole des Mines d'Albi, UMR CNRS 5302, Centre Rapsodee, F-81013 Albi, France
}

Supporting Information

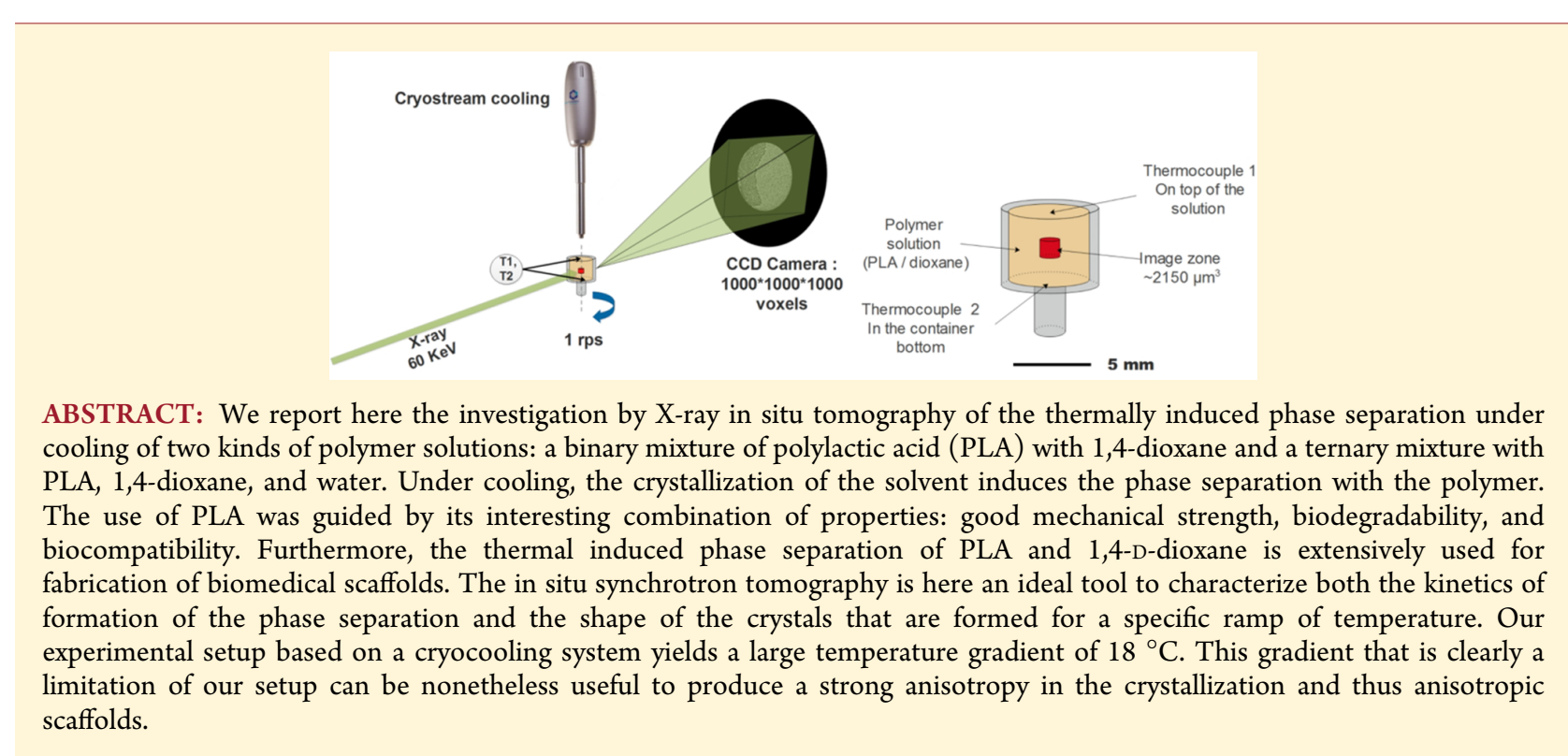

\section{INTRODUCTION}

Thermally induced phase separation (TIPS) invented in the late 1970s has become a key technique for producing porous polymer matrices after extraction of the solvent. ${ }^{1,2}$ The TIPS process is based on the principle that a homogeneous solution of a polymer dissolved in a good solvent can undergo a phase separation by cooling and can consequently cause solution saturation which leads to polymer precipitation, followed by polymer structuration as a microcellular foam. The cooling of the polymer solution may trigger the phase separation depending on how the crystallization temperature of the solvent is approached. Traditionally two kinds of phase separation could be induced for polymer scaffold production by TIPS technology. The first one is the solid-liquid phase separation. It requires the use of a polymer and a solvent having close solubility parameters. Preferably, the solvent must have a high crystallization temperature. In this case, the separation is guided by the fact that when the solvent reaches its freezing point, it undergoes a phase transformation from a liquid to a solid crystalline phase. When the formed crystals are large enough in size, they cannot be sustained anymore to remain in contact with the polymer. A polymer-rich phase then becomes separated from a crystallized solvent-rich phase. Thus, the shaping of the ultimate polymer network is governed by the growth of the solvent crystals. The second one is the liquid-liquid phase separation: it occurs when the cloud point temperature is superior to the temperature of freezing. Thereby, the removal of the thermal energy from the solution causes the separation in two liquid phases: a polymer-rich phase and a polymer-lean phase. ${ }^{3}$

After the phase separation of the polymer and the solvent is achieved, a porous structure can be revealed by solvent 
extraction. By tuning different parameters such as the polymer/ solvent nature, the polymer concentration, the quench temperature, the quenching rate or the solvent, different pathways can be achieved. This in turn yields different scaffold morphologies. Although TIPS has been used for a long time in industry to produce porous polymer scaffolds ${ }^{4-7}$ and membranes, ${ }^{3,8-11}$ it is rather amazing that no in situ experiment has been reported on the kinetics of formation of such porous architectures. So far, classical investigation techniques (like scanning electron microscopy, SEM) are usually utilized to evidence the porous structure, but this is done post-mortem. Another drawback of SEM is its inability to properly characterize the overall complex porous structures. ${ }^{12}$ It is subsequently still very challenging to monitor in situ the formation of the phase separated regions during the TIPS process in order to unveil the role of the different parameters that can affect the pore formation. In particular, in situ studies offer the possibility to follow the kinetics of crystal growth by monitoring in three dimensions (3D) the evolution of the crystal growth. It is indeed possible, with the advance of third generation synchrotron sources, to obtain in situ images of phase transformations by X-ray microtomography in 3D with sufficient spatial and time resolution. ${ }^{13-16}$ With the use of 3D microtomography, one can thus expect that the key parameters inducing the pore morphology (open/closed pores) and their anisotropy could be accessed at the early stages of the TIPS process. So far, the only technique that can provide such information is in situ microtomography at synchrotron facilities. The 3D image of a relatively large volume (1-6 $\mu \mathrm{m}^{3}$ ) can be obtained within less than a second with a resolution spanning the characteristic length scales of the porosity that can be encountered in foams, i.e., ranging from the sub-micrometer scale to a few microns depending on the experimental setup. It is nondestructive and provides $3 \mathrm{D}$ data on material morphology.

In this paper, we have investigated by X-ray tomography the phase separation under cooling of two kinds of polymer solutions: a binary mixture of polylactic acid (PLA) and 1,4dioxane and a ternary mixture of PLA, 1,4-dioxane, and water. The use of PLA was guided by its interesting combination of properties: good mechanical strength, biodegradability, and biocompatibility. Furthermore, this polymer has been extensively used for fabrication of scaffolds for drug delivery in tissue engineering. ${ }^{17-19} \mathrm{~A}$ specific in situ device was designed to follow the phase separation under cooling and especially the crystal growth. Moreover, ex-situ foams were also analyzed for the sake of comparison.

\section{EXPERIMENTAL SECTION}

2.1. Materials. The PLA polymer used was a poly-D-lactic acid (PDLA) type. It was supplied by Natureplast (PDLA001) and contains more than $99 \%$ of D-isomer, making it particularly crystalline. The crystallinity rate was measured by DRX at $24 \%$ on the raw material. Its molar mass $\left(M_{\mathrm{w}}\right)$ measured by steric exclusion chromatography was found to be $47000 \mathrm{~g} / \mathrm{mol}^{-1}$ with an index of polydispersity (PI) equal to 1.36. 1,4-Dioxane (ACS reagent, >99\%) was bought from Sigma-Aldrich. Milli-Q water was added to provoke the liquid-liquid phase separation.

1,4-Dioxane was chosen as the solvent due to its affinity with PLA. Indeed, its Hansen solubility parameter $\left(\delta=20.5 \mathrm{MPa}^{1 / 2}\right)$ is close to the one of PLA $\left(\delta=21.9 \mathrm{MPa}^{1 / 2}\right)$. The proximity of these two values together with their somewhat similar contribution for the polar and dispersion coefficients and a weaker hydrogen bonding coefficient makes 1,4-dioxane a good solvent of PLA. In addition, the melting temperature of 1 ,4-dioxane $\left(T_{\mathrm{m}} \approx 11.5^{\circ} \mathrm{C}\right.$ see Table 1$)$ is the highest compared to other solvents, and it is thus easier to work with this

Table 1. Solubility Parameters of Different Components Together with Their Freezing Temperature ${ }^{20}$

\begin{tabular}{lccccc}
\multicolumn{1}{c}{ component } & $\begin{array}{c}\delta \mathrm{p}^{1 / 2} \\
\left(\mathrm{MPa}^{1 / 2}\right)\end{array}$ & $\begin{array}{c}\delta \mathrm{h} \\
\left(\mathrm{MPa}^{1 / 2}\right)\end{array}$ & $\begin{array}{c}\delta \mathrm{d} \\
\left(\mathrm{MPa}^{1 / 2}\right)\end{array}$ & $\begin{array}{c}\delta \mathrm{t} \\
\left(\mathrm{MPa}^{1 / 2}\right)\end{array}$ & $\begin{array}{c}T_{\mathrm{m}} \\
\left({ }^{\circ} \mathrm{C}\right)\end{array}$ \\
poly(lactic acid) & 9.9 & 6 & 18.6 & 21.9 & \\
chloroform & 3.1 & 5.7 & 17.8 & 18.9 & -64 \\
dichloromethane & 6.3 & 6.1 & 18.2 & 20.2 & -95 \\
dichloroethane & 7.8 & 3.0 & 16.5 & 18.5 & -35 \\
1,4-dioxane & 7.4 & 1.8 & 19.0 & 20.5 & 11.5 \\
water & & & & 48 & 0 \\
\hline
\end{tabular}

solvent than the others when it comes to dissolving the PLA. Note that the freezing temperature of 1,4-dioxane was also measured by differential scanning calorimetry (DSC) and was found to be close to $T_{\mathrm{c}} \approx 0{ }^{\circ} \mathrm{C}$ at $-1{ }^{\circ} \mathrm{C} / \mathrm{min}$ cooling speed. This temperature markedly differs from the $T_{\mathrm{m}}$, measured by DSC to $11.5{ }^{\circ} \mathrm{C}$.

For the ternary mixture, water was chosen as the additional nonsolvent of PLA. The main objective for adding a small amount of water in the solution was to modify the equilibrium by lowering the polymer/solvent interaction. In the case of the binary mixture, the good compatibility between the two components favors during the cooling the crystallization of the solvent that expels the polymer chains and consequently causes the phase separation. So, the process is a solid-liquid phase separation, whereas when water is added, a liquid-liquid phase separation dominates the process. This point is very important because scaffolds obtained by these two ways can be drastically different.

2.2. Solution Preparation. Solutions of PDLA in 1,4-dioxane were prepared by dissolving PDLA pellets in 1,4-dioxane under stirring at $300 \mathrm{rpm}$ and $50{ }^{\circ} \mathrm{C}$. The amount of PDLA could be tuned between 5 and $10 \%$ in weight. In the case of ternary mixtures, water was added after complete dissolution of the polymer in the 1,4dioxane. Three PDLA binary mixtures were prepared with weight percentage concentrations of $5 \% \mathrm{w} / \mathrm{w}$ and $10 \% \mathrm{w} / \mathrm{w}$. One ternary mixture was prepared with weight percentage concentrations and 7.5/ $80.5 / 12 \% \mathrm{w} / \mathrm{w}$.

2.3. Experimental Set Up. To follow the phase separation under cooling yielding the formation of a PDLA scaffold, a special device was designed and mounted on the ID 19 microtomography beamline at the European Synchrotron Radiation Facility (ESRF). The device (shown in Figure 1) was composed of a cooling system with an Oxford Cryostream device able to blow a nitrogen flow at regulated temperature and of an instrumented sample holder in poly ether ether ketone (PEEK). The sample holder was equipped with two thermocouples located at the top and bottom of the peek container, thus enabling the temperature gradient in the sample to be measured. Note that this setup was chosen because of its simplicity. The PEEK cell is transparent to X-rays, and the absence of any plugging cables ensured the free rotation of the sample during the data collection. Yet this advantage was counterbalanced by the presence of a thermal gradient due to the flow of cold nitrogen. More fancy cooling systems should be envisaged in the future to prevent the thermal gradient.

In order to perform the experiments, a PCO dimax detector was used in which we selected a ROI $1008^{*} 1008$ pixels for in situ experiments and $2016^{*} 2016$ pixels for ex-situ experiments. The pixel size was $1.4 \mu \mathrm{m}$ with an exposure time of $0.001 \mathrm{~s}$. All measurements were performed with a pink beam centered at energy of $68 \mathrm{keV}$. The sample to detector distance was fixed at $460 \mathrm{~mm} \mathrm{~A}$ full in situ tomograph consisted of a 3D image of $1008 * 1008 * 1008$ voxels.

2.4. Choice of the Temperature Range. At the beginning of any tomography experiments, a small amount $(0.3 \mathrm{~mL})$ of the polymer solution was poured into the PEEK container. The gun of the cryostream regulated at $250 \mathrm{~K}$ was placed above the solution immediately prompting the temperature decrease. Tomography scans were started about $2 \mathrm{~min}$ later. The duration of a scan was fixed to $1 \mathrm{~s}$. 

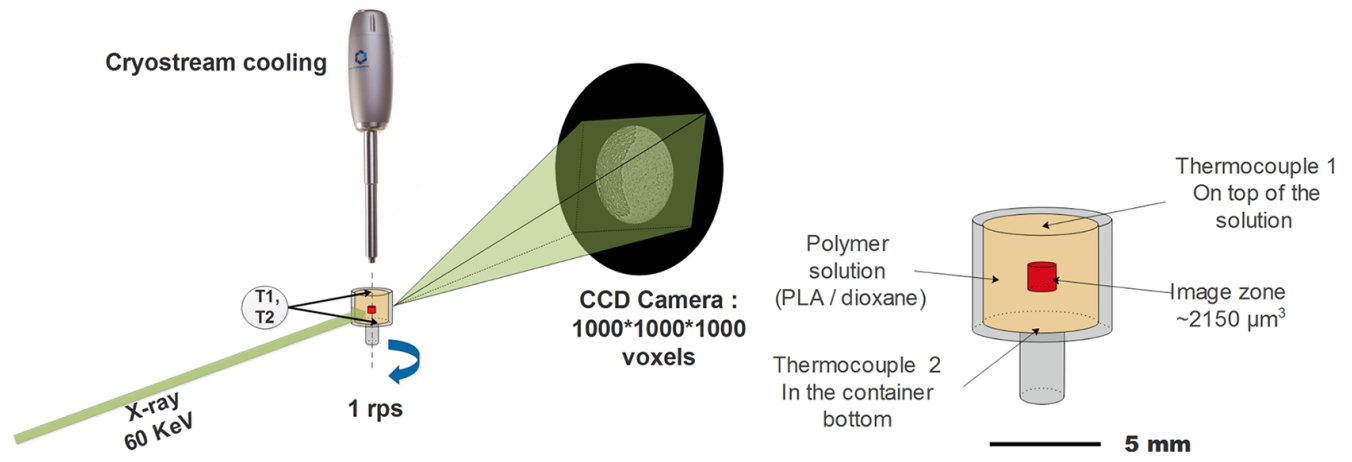

Figure 1. Schematic illustration of the device used to in situ experiments (left) the instrumented sample holder (right).

Scans were continuously recorded every $25 \mathrm{~s}$ to observe the phase separation mechanisms caused by cooling. The choice of the cryostream temperature was guided by trial and errors experiments during which visible phase separation consistent with the resolution of the instrument was tested. For instance, it was not possible to observe any changes when the cryostream temperature was set to $220 \mathrm{~K}$. We interpreted this result as a consequence of the formation of very small crystallites that were not measurable with a pixel size of $1.4 \mu \mathrm{m}$.

In order to carry out the tomographic experiment in the right range of temperature, the freezing temperature of the PDLA/1,4-dioxane binary mixture was analyzed by DSC and visual turbidimetry. ${ }^{21}$ By DSC a small amount of solution was dropped, and a cooling ramp was set from $25{ }^{\circ} \mathrm{C}$ to $-20{ }^{\circ} \mathrm{C}$ at $-1{ }^{\circ} \mathrm{C} / \mathrm{min}$ speed. The onset crystallization temperature was taken. DSC measurements were carried out on a Q20 from TA Instruments coupled with a nitrogen cooling system, whereas the turbidimetry measurement were carried out in a cooling water bath. Some vessels with organic solutions were placed into the bath, and a cooling ramp was set from $25{ }^{\circ} \mathrm{C}$ to -10 ${ }^{\circ} \mathrm{C}$ at $-0.1{ }^{\circ} \mathrm{C} / \mathrm{min}$ speed.

The results are shown in Figure 2. The cooling rate has a huge influence on the value of the solution freezing point. A faster cooling

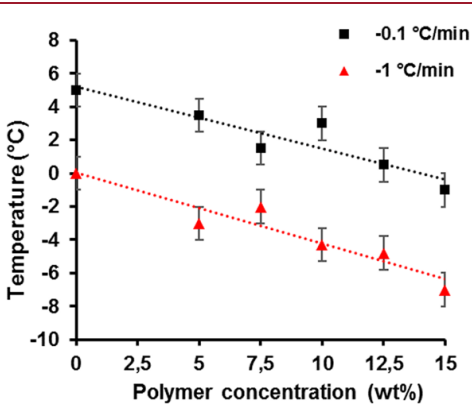

Figure 2. Crystallization temperature of the binary mixture PDLA/ 1,4-dioxane as a function of PDLA concentration. The black curve $\left(-0.1{ }^{\circ} \mathrm{C} / \mathrm{min}\right)$ was determined by turbidimetry and the red curve $\left(-1{ }^{\circ} \mathrm{C} / \mathrm{min}\right)$ using a DSC apparatus.

speed decreases the freezing temperature. Moreover, this temperature decreases also with the concentration of polymer. This is a wellknown effect observed when impurities are added to a solvent.

To ensure the crystallization of the binary mixture, we chose to impose a temperature around $-10{ }^{\circ} \mathrm{C}$ at the top of the sample holder. This corresponded to a regulation of about $-23{ }^{\circ} \mathrm{C}$ of the nitrogen flow places at about $1 \mathrm{~cm}$ above the sample. Note that for a concentration of 5\% of PDLA, the crystallization is expected to start between $+4{ }^{\circ} \mathrm{C}$ and $-3{ }^{\circ} \mathrm{C}$ as deduced from Figure 2 . We therefore could assume that if the top of the cell was at $T=-10{ }^{\circ} \mathrm{C}$ the crystallization should definitely occur in the top of the cell. The proximity of this temperature with the one of the crystallization temperature of the solution ensures that the crystals to be formed will be large enough to be measurable with our instrumental resolution (estimated to be of the order of $3 \mu \mathrm{m}$ ). Note that a quench at a much lower temperature will produce much smaller crystals that will be impossible to detect with the instrumental resolution or even no phase separation at all.

2.5. Scaffolds Characterization. Some tomographs of dry scaffolds have been measured. These data have been investigated in comparison with in situ samples and with previous characterization from SEM.

2.5.1. Scaffold Porosity and Density Measurements. The global scaffold porosity $(\varepsilon)$ was determined by indirect measurement of the scaffold density from the following equation.

$$
\varepsilon(\%)=1-\frac{\rho_{\text {scaffold }}}{\rho_{\text {polymer }}}
$$

The scaffold density measurement of scaffolds was assessed by the water displacement method. The whole sample was first weighed dry, and second weighed in a $25 \mathrm{~mL}$ measuring cylinder filled with water. Because of the buoyancy of the scaffolds, a metal cylinder with known volume was used to maintain the scaffold at the bottom of the measuring cylinder. In this method, it was assumed that the water did not penetrate within the sample because of the small size of external pores and the short time of immersion.

2.5.2. SEM Observations. Scaffolds morphology was observed by SEM on a JEOL 6301F (JEOL Paris, France). A thin layer was cut in the center of the scaffolds. This sample was coated with a thin layer of platinum by sputtering with a high vacuum coater (Leica EM ECA600, Leica, France). Images were captured at a $3 \mathrm{kV}$ acceleration voltage in the secondary electron mode and a working distance of 20 $\mathrm{mm}$.

The pore size was determined by image analysis using Image ${ }^{22}$ freeware on the SEM micrographs. The pore sizes were obtained by measuring at least 30 pores by samples, except for the sample from ternary mixture because it has very large pores. The measurement took into account for pore size the small diameter.

2.5.3. 3D Analysis. From the tomographs' pore size and of the PDLA wall thickness was carried out using Bone ${ }^{23},{ }^{23}$ a free plugin for the open source software ImageJ. The "Thickness" plugin was used for these measurements. This program is based on Hildebrand and Rüegsegger works ${ }^{24}$ about local thickness assessment and develop by Dougherty and Kunzelman. ${ }^{25}$

\section{RESULTS AND DISCUSSION}

3.1. Temperature Gradient. Before discussing in detail the results of this experiment, it is important to understand that the cryocooling gas setup that was used did not enable an homogeneous vertical distribution of the temperature inside the cell to be produced even though the cell height was quite small $(h<4 \mathrm{~mm})$. Figure 3 shows the evolution of the temperature measured with the two thermocouples at the top and bottom of the cell as a function of time during a scan. The 


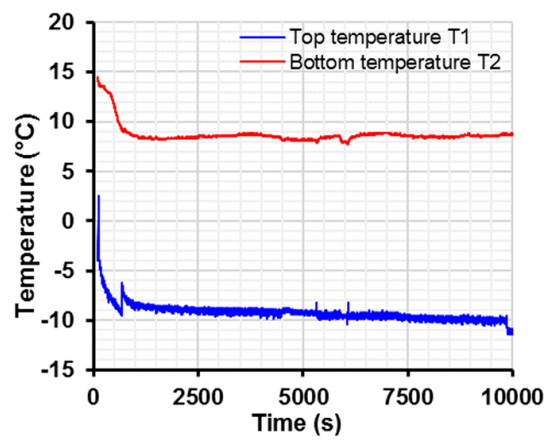

Figure 3. Evolution of the temperature at the top and bottom of the cell as a function of time during a scan (the nitrogen flow was regulated at $250 \mathrm{~K})$.

steady state was reached after about $1000 \mathrm{~s}$. The nitrogen flow was regulated at $250 \mathrm{~K}$, and the temperature of the top part of the cell was close to $-10{ }^{\circ} \mathrm{C}$, while the bottom one was close to $8{ }^{\circ} \mathrm{C}$. The temperature gradient was thus about $18{ }^{\circ} \mathrm{C}$, the bottom temperature being higher than the freezing point of the solvent. As the cell height was $4 \mathrm{~mm}$, the temperature gradient was estimated to be equal to $4.5{ }^{\circ} \mathrm{C} / \mathrm{mm}$. Due to the presence of a temperature gradient, the onset of crystallization and phase separation did not occur at the same time and at different heights in the sample.

3.2. Solid-Liquid Phase Separation. Figure 4 that shows $2 \mathrm{D}$ vertical slices extracted from the $3 \mathrm{D}$ tomograms probe the progression of the crystallization front in PDLA/1,4dioxane solution at $5 / 95 \% \mathrm{w} / \mathrm{w}$. When time passes by, dark regions appear on the slices. At $1250 \mathrm{~s}$, a large number of dark particles are already formed at the top of the image. These regions have grown both laterally and vertically up to this time. After $1250 \mathrm{~s}$, the growth becomes more prominent along the vertical. These slices are obtained by phase contrast imaging as the ones issued from absorption only were too poor to be properly analyzed. This definitely shows the superiority of the phase contrast imaging for such an analysis. At $t=0 \mathrm{~s}$, the slice is uniformly gray showing that PDLA is homogeneously dissolved in the 1,4-dioxane. The darker regions correspond to 1,4-dioxane crystals, whereas the brighter ones stand for the binary PDLA/1,4-dioxane solution. Note that the density of PDLA is expected to be close to 1.3 , while the one of liquid 1,4-dioxane is close to 1 , so the darker region is attributed to pure 1,4-dioxane crystals that should be less dense than the PDLA/1,4-dioxane solution. It is obvious from Figure 4 that the crystallization of 1,4-dioxane starts at the top surface and progresses downward across the solution as a function of time. After about $1250 \mathrm{~s}$, the growth is mainly directed downward as crystals are touching each other in-plane. The growth is thus hampered in plane by percolation of neighboring 1,4-dioxane crystals.

To fully appreciate the anisotropy of the growth process, we have extracted as a function of time $2 \mathrm{D}$ slices at three different heights below the surface in order to monitor the in-plane progression of crystallization (see Figure 5). It is obvious from these images that the growth is quite slow and more importantly does not occur similarly at different heights. In slice A of Figure 5 which the closest to the top surface, the particles steadily grow and rapidly invade a large part of the slice as seen at $7500 \mathrm{~s}$. At a deeper altitude in the cell (see slices B and C of Figure 5), it is clear that, even after $5000 \mathrm{~s}$,

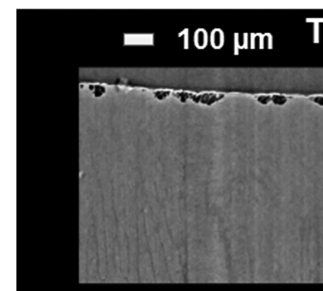

$t=0 s$

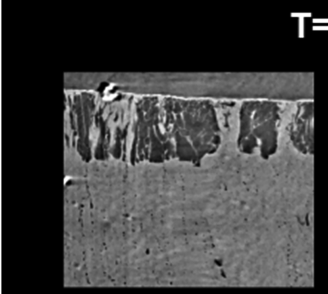

$t=3750 s$

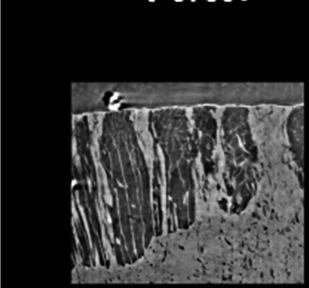

$\mathrm{t}=7500 \mathrm{~s}$

\section{$\mathrm{T}=-8,5^{\circ} \mathrm{C}$}

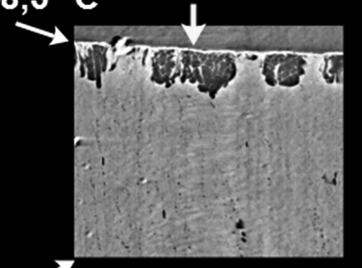

$\mathrm{t}=1250 \mathrm{~s}$

$\mathrm{T}=-4^{\circ} \mathrm{C}$

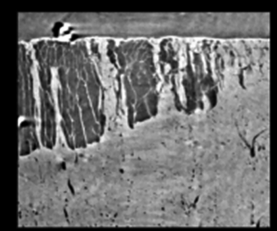

$\mathrm{t}=5000 \mathrm{~s}$

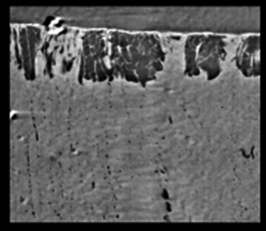

$\mathrm{t}=2500 \mathrm{~s}$

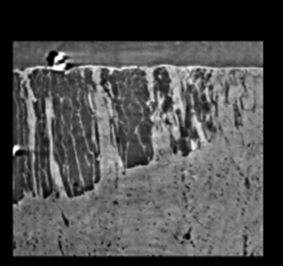

$\mathrm{t}=6250 \mathrm{~s}$

Figure 4. Vertical cuts carried out in the 3D tomograms showing the progression of crystallization front across the solution (PDLA/1,4-dioxane 5/ $95 \% \mathrm{w} / \mathrm{w}$ ) as a function of time (in seconds). 


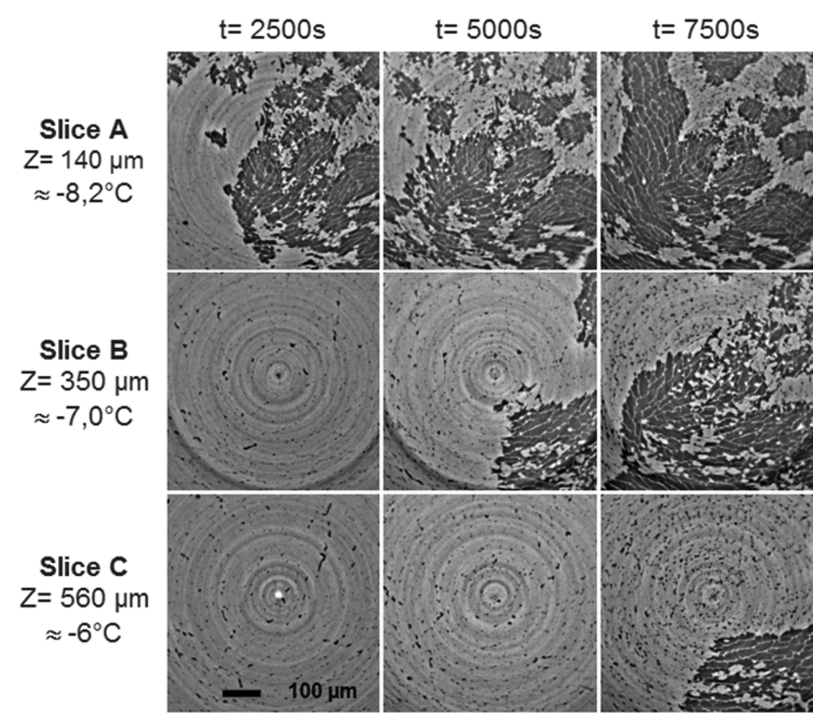

Figure 5. Horizontal cuts at different heights from the top of the solution (PDLA/1,4-dioxane $5 / 95 \% \mathrm{w} / \mathrm{w}$ ) at 2500,5000 , and $7500 \mathrm{~s}$.

the crystallization has barely begun. Note again that the images obtained here are limited by the resolution of the instrument with a voxel size of $1.4 \times 1.4 \times 1.4 \mu \mathrm{m}^{3}$. Any feature below this threshold is therefore smeared out. This statement is valid for both the crystals forming and even more for the PDLA minority phase that occupies a small fraction of the final volume. This phase is the one that will remain after solvent extraction. The instrumental resolution is also a limiting factor for exploring the evolution of the size of the crystallites upon a quench at very low temperature, as in this case the crystallites will be too small to be detected. Here, the results correspond to the top part of the cell being at about $-10{ }^{\circ} \mathrm{C}$ in the steady state. To confirm the previous statement, all measurements made with a top temperature of $-50{ }^{\circ} \mathrm{C}$ failed as the crystallites were likely too small. One of the striking features on these slices is the nonergodicity of the different regions of the slices. Indeed, from a statistical viewpoint, some regions are crystalline free, while others are overcrowded. It can be argued that this could result from a temperature gradient inside the cell and by the presence of nucleation germs close to the wall of the cells.

More realistic views of the evolution of the crystallization are obtained in 3D plots of the monitored zone. These 3D graphs, shown in Figure 6, are very informative as they again evidence that the crystals grow from the top surface toward the bottom part of the cell due to the thermal gradient. At the very top, a clear boundary separates some frost due to the cooling cryostream and the crystalline phase. Below the crystalline phase, a somewhat homogeneous medium corresponds to the liquid solution which has not yet crystallized. Indeed, turbidimetry measurements (Figure 2) have clearly established that, at a concentration of $5 \% \mathrm{w} / \mathrm{w}$ of polymer in 1,4-dioxane, the crystallization temperature is on the order of $3.5^{\circ} \mathrm{C}$. This indicates that the crystallization would be extremely difficult in the bottom part of the cell as evidenced in the tomograms. Note that this is a serious challenge in $3 \mathrm{D}$ tomography as the presence of a liquid state inside the cell on top of which are lying crystals may induce some movements of the crystalline phase during the rotation. This is why we used the shortest time of $1 \mathrm{~s}$ to carry out a full scan. Yet we found out that, even

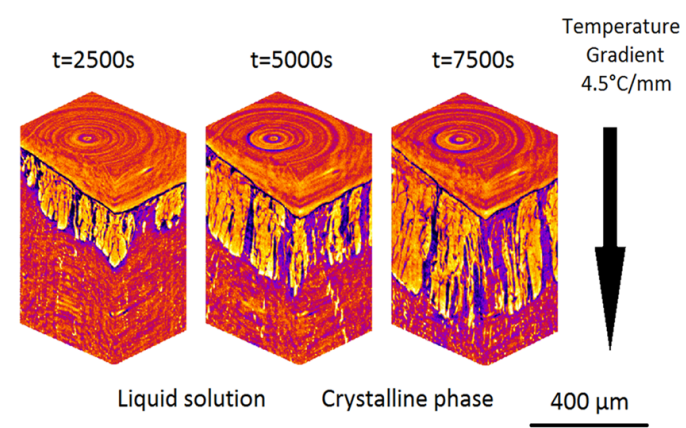

Figure 6. 3D representation of the phase separation for a PDLA/1,4dioxane solution at different times showing the progression of the crystalline phase along the temperature gradient. On the left, the arrows indicate the liquid solution, while on the right, the arrow indicates the crystalline phase.

though we took extreme care to avoid this problem, some regions were moving. This was clearly the case after $7500 \mathrm{~s}$ so that we have reported here the analysis up to this time.

A $3 \mathrm{D}$ view of a selected crystal inside the cell was then isolated to explore its evolution versus time. Note that it was difficult to extract such a crystal, as in many areas the crystals formed at the beginning of the process grew laterally and merged with each other to produce a continuous network. As shown in Figure 7, one can easily observe that this crystal is mainly growing along the thermal gradient up to a certain time

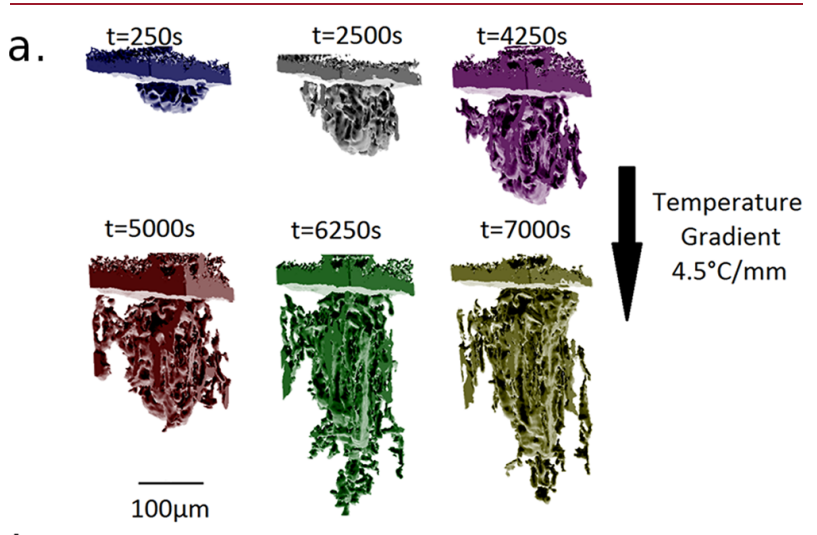

b.

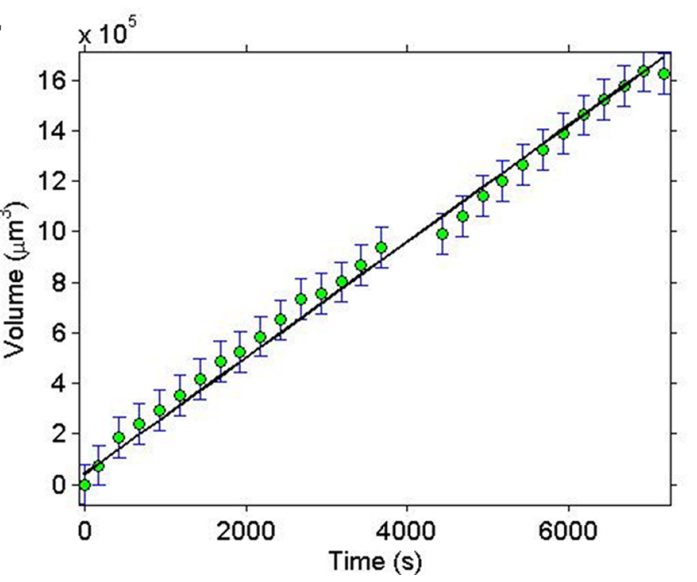

Figure 7. (a) 3D representation of an isolated crystal of 1,4-dioxane as a function of time; (b) evolution of the volume of this crystal versus time showing a linear behavior. 
where the growth is over. We can estimate in this case how the volume of the crystal changes as a function of time.

A careful analysis of the crystalline phase showed that most of the crystalline phase was actually constituted of a continuous network of small crystals touching each others. However, it was possible to isolate a few crystals that were well separated from the remaining continuous network, and these crystals were used to explore the evolution of their volume versus time. As shown in the 3D view of Figure 7, 1,4-dioxane crystals are mainly growing along the thermal gradient up to a certain time where the growth is over. Then, the volume of the crystal can be estimated as a function of time.

We evidence in Figure $7 \mathrm{~b}$ that the volume increases linearly as a function of time up to a certain time $(t<7500 \mathrm{~s})$ beyond which it remains constant. By monitoring the crystal height versus time, a linear behavior was also observed. This clearly shows that the surface of the selected crystal was almost constant during the growth and that the growth was mainly directed along the temperature gradient. Similar experiments conducted with another concentration of $10 \% \mathrm{w} / \mathrm{w}$ of PDLA in 1,4-dioxane are presented in the Supporting Information S1.

3.3. Liquid-Liquid Phase Separation. The phase separation was also studied in situ for the ternary mixture for which the composition PDLA/1,4-dioxane/ $\mathrm{H}_{2} \mathrm{O}$ is 7.5/80.5/ $12 \% \mathrm{w} / \mathrm{w}$. Some selected slices of the $3 \mathrm{D}$ matrix are shown in Figure 8 . In comparison with the binary mixture, a radically

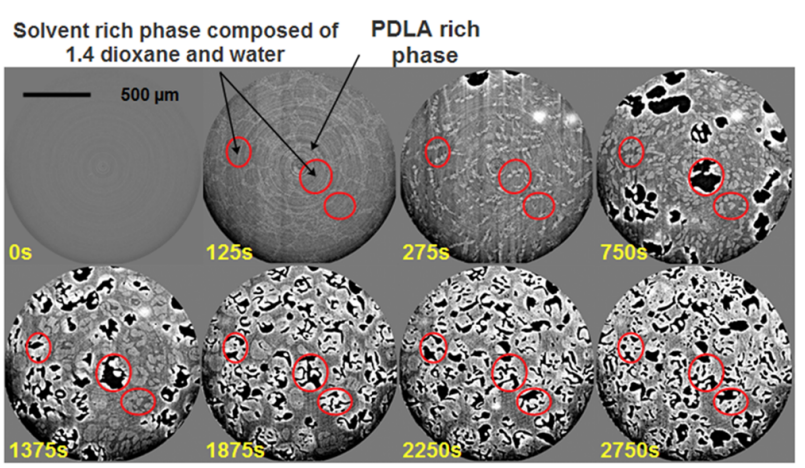

Figure 8. Slices taken at $z=280 \mu \mathrm{m}$ from the top surface of the cell showing the phase separation mechanism of a ternary solution.

different scenario is observed. After only $125 \mathrm{~s}$ of cooling, a structure similar to a scaffold produced by homogeneous quench is observed. In fact, for the same polymer concentration, the phase separation of the ternary solution occurred at a higher temperature than in binary solution. The cloud point of the ternary mixture has been measured by turbidimetry at $11^{\circ} \mathrm{C}$ for a cooling speed of $1{ }^{\circ} \mathrm{C} / \mathrm{min}$ (not shown), whereas at the temperature at this height and time is estimated at $-1.5^{\circ} \mathrm{C}$. We here have two to three levels of gray scale in the images: a black portion, a gray one, and a white one. At $125 \mathrm{~s}$, the dark gray, which is less dense than the light gray portion, is likely due to a liquid phase composed of 1,4dioxane and water (density close to 1 ). The light gray one would be a phase enriched in PDLA (density close to 1.3). At a longer time, black regions evidence the appearance of an even less dense phase that we attribute to ice formation (density 0.85 ). DSC measurements showed that the 1,4-dioxane/water mixture crystallized at a lower temperature (below $-12{ }^{\circ} \mathrm{C}$ ) than the pure 1,4-dioxane (below $0{ }^{\circ} \mathrm{C}$ ). The hypothesis is that the proximity of the temperature in the cell with the crystallization temperatures of the two compounds should explain the instability of these two phases. It should be noted that the polymer-rich phase does not appear to be affected by the phenomena of crystallization. Thus, the images obtained allowed the first visual evidence of the shaping of scaffolds by liquid-liquid phase separation to be produced. However, it is difficult to go further in the analysis because, in the early stages of phase separation, there is a lack of contrast and then artifacts (characterized by white boundaries around the black regions) appear because of density differences.

3.4. Ex Situ Scaffold Analysis. The results reported in the previous section are quite interesting to in situ unveil the progression of the phase separation. Yet the thermal gradient was a serious handicap to fully understand how scaffolds could be made when materials are made by uniform quenches. This is why we have also performed tomography on samples for which the quench was uniform and for which the solvent was removed with supercritical $\mathrm{CO}_{2}$ after phase separation.

The 3D tomograms of a binary mixture (shown in Figure 9) clearly show that the porosity was better spatially distributed.

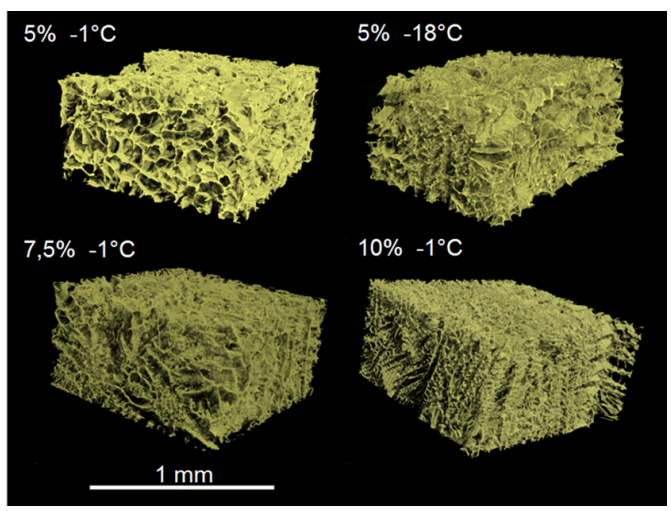

Figure 9. 3D tomograms of the PDLA scaffolds after solvent extraction for different quenching temperatures and different PDLA concentrations.

Pores were smaller at a high concentration of PDLA than at a low one, as expected from the density. The full analysis of the pore size and of the PDLA wall thickness was carried out using BoneJ, a free plugin for the open source software ImageJ. Results confirm this statement and show that the wall thickness becomes a bit bigger if the concentration of the polymer increases (see Table 2). With the exception of the sample produced from the ternary solution, results extracted from 3D tomograms are in agreement with the porosity measured by water pycnometry. The difference in the porosity results could be easily explained by the presence of pores below the tomograph resolution about $1.4 \mu \mathrm{m}$ in size (see Figure 10). Furthermore, scaffolds obtained by a solid-liquid separation showed a porosity decrease when the concentration of the polymer increased. The difference between the pore size extracted from tomograms and measured on SEM pictures has several explanations: first of all the analysis of 3D takes into account a bigger number of pores in a large sample volume compared to one or two slices in one sample. Second, measuring the $3 \mathrm{D}$ element with $2 \mathrm{D}$ representation inevitably induces a measurement error when the objects are not perfectly isotropic. Then, more human action is taken for measurements, the more the sources of errors increase. 


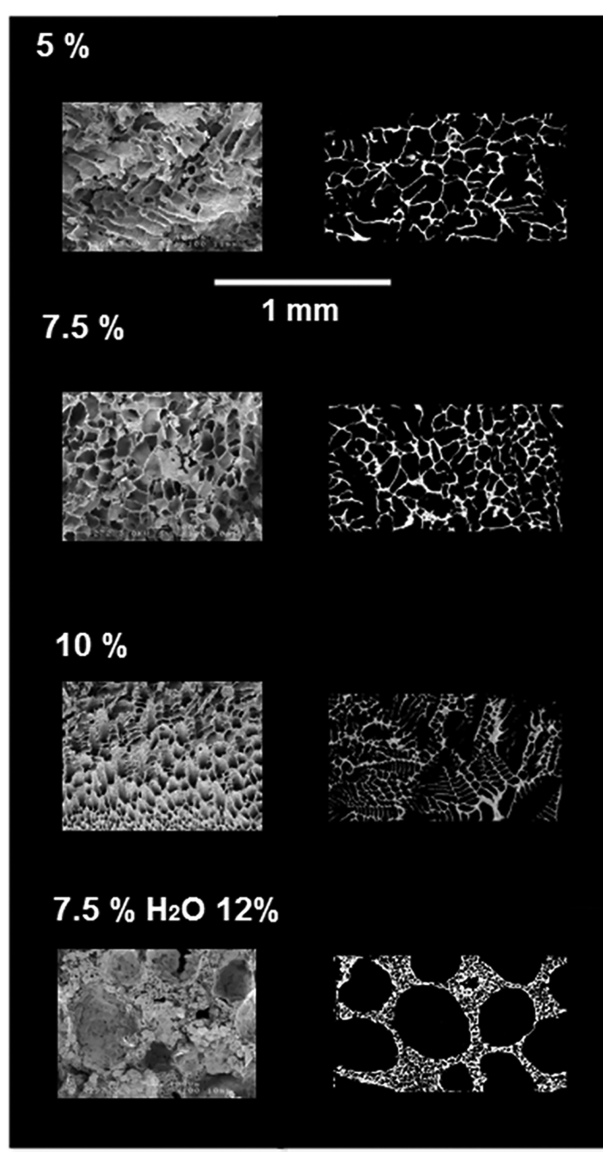

Figure 10. Comparison of SEM images (left) and slice extracted from 3D tomograph (right) taken in the same scaffold.

However, even if the $2 \mathrm{D}$ measurement is not accurate, the order of magnitude is correct. Thus, we can conclude in both cases that the increase of the polymer content in binary solutions decreases the pore diameter. In addition, at the same concentration the decrease of the temperature decreases the pore size. The relation between experimental parameters and scaffold properties is deeply described in our previous paper about the development of the TIPS process. ${ }^{26}$

\section{CONCLUSION}

We have shown that in situ tomography is a key technique to understand the mechanism and kinetics of phase separation under cooling of PDLA solutions in 1,4-dioxane. The developed methodology is particularly efficient to monitor the solid-liquid phase separation and to follow the progression of the crystallization front in TIPS experiments. Yet a major drawback of our setup was the existence of a very large thermal gradient which markedly differed from the uniform quench traditionally utilized to produce polymer scaffolds by TIPS. The existence of such a gradient was nevertheless of great interest to produce elongated 1,4-dioxane crystals in the direction of the thermal gradient. In this sense, the method we have used here is quite similar to the one used for producing oriented membranes by TIPS. So far, to the best of our knowledge, this is the first experiment of this kind. The method still suffers from a lack of spatial resolution (pixel size $>1.4 \mu \mathrm{m}$ ) to determine in a more precise way the formation of crystallites at lower temperatures. Nonetheless, it was possible to show that 3D tomography could be used to measure the crystal growth and to unravel the porosity of PDLA scaffolds when the solvent was extracted.

\section{ASSOCIATED CONTENT}

\section{Supporting Information}

The Supporting Information is available free of charge on the ACS Publications website at DOI: 10.1021/acs.cgd.8b01309.

Experiments similar to the ones carried out for $5 \%$ of PDLA in 1,4-dioxane were conducted with another concentration of $10 \%$. The results are reported (PDF)

\section{AUTHOR INFORMATION}

\section{Corresponding Author}

*E-mail: gibaud@univ-lemans.fr.

\section{ORCID $\odot$}

Thomas Beuvier: 0000-0002-4458-1658

Alain Gibaud: 0000-0002-7777-6427

\section{Present Address}

"II CRCINA, INSERM, Université de Nantes, Université d'Angers, Angers, France; INSERM U1232, Team-17, GLIAD - Design and Application of Innovative Local treatments in Glioblastoma, Université d'Angers, IBS - CHU, 4 Rue Larrey, 49933.

\section{Notes}

The authors declare no competing financial interest.

\section{ACKNOWLEDGMENTS}

The authors are grateful for the financial support from the MATIERES Project and the "Région Pays de la Loire". The authors thank the ESRF for providing beamtime at the ID19 beamline. The authors are indebted to J-P. Valade for his technical support during the preparation of the tomography experiments at the ID19 beamline. The authors thank the SCIAM ("Service Commun d'Imagerie et d'Analyse Micro-

Table 2. Evolution of the Pore Diameter, Wall Thickness, and Porosity of PDLA Samples Solutions in 1,4-Dioxane Quenched

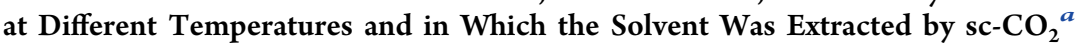

\begin{tabular}{lcccccc}
\multicolumn{1}{c}{ sample } & $\begin{array}{c}\text { temperature } \\
\left({ }^{\circ} \mathrm{C}\right)\end{array}$ & $\begin{array}{c}\text { pore size }(\mu \mathrm{m}) \text { from 3D } \\
\text { mean } \pm \text { STD }\end{array}$ & $\begin{array}{c}\text { pore size }(\mu \mathrm{m}) \text { from SEM } \\
\text { mean } \pm \text { STD }\end{array}$ & $\begin{array}{c}\text { wall thickness }(\mu \mathrm{m}) \\
\text { mean } \pm \text { STD }\end{array}$ & $\begin{array}{c}\text { porosity }(\%) \\
\text { from 3D }\end{array}$ & $\begin{array}{c}\text { global porosity }(\%) \\
\text { from pycno }\end{array}$ \\
PDLA 5\% & -1 & $67 \pm 37$ & $63 \pm 13$ & $7.9 \pm 3.0$ & 8.9 \\
PDLA 5\% & -18 & $47 \pm 41$ & $54 \pm 15$ & $7.4 \pm 2.4$ & $87.8 \pm 2.9$ \\
PDLA 7.5\% & -1 & $46 \pm 19$ & $59 \pm 12$ & $9.4 \pm 3.7$ & $87.0 \pm 3.0$ \\
PDLA 10\% & -1 & $40 \pm 22$ & $35 \pm 11$ & $8.8 \pm 3.0$ & 80 \\
PDLA 7.5\% $\mathrm{H}_{2} \mathrm{O}$ & -1 & $71 \pm 40$ & $191 \pm 75$ & $7.6 \pm 2.4$ & 80.8 \\
12\% & & & & & 51.2
\end{tabular}

${ }^{a}$ These parameters were obtained from the 3D tomograms (Figure 10). 
scopique", University of Angers) for scanning electron microscopy images.

\section{REFERENCES}

(1) van Aartsen, J. J. Theoretical Observations on Spinodal Decomposition of Polymer Solutions. Eur. Polym. J. 1970, 6, 919924.

(2) Bank, M.; Leffingwell, J.; Thies, C. Thermally Induced Phase Separation of Polystyrene-Poly(Vinyl Methyl Ether) Mixtures. J. Polym. Sci. Part -2 Polym. Phys. 1972, 10, 1097-1109.

(3) van de Witte, P.; Dijkstra, P. J.; van den Berg, J. W. A.; Feijen, J. Phase Separation Processes in Polymer Solutions in Relation to Membrane Formation. J. Membr. Sci. 1996, 117, 1-31.

(4) Molladavoodi, S.; Gorbet, M.; Medley, J.; Ju Kwon, H. Investigation of Microstructure, Mechanical Properties and Cellular Viability of Poly(L-Lactic Acid) Tissue Engineering Scaffolds Prepared by Different Thermally Induced Phase Separation Protocols. J. Mech. Behav. Biomed. Mater. 2013, 17, 186-197.

(5) Ho, M.-H.; Kuo, P.-Y.; Hsieh, H.-J.; Hsien, T.-Y.; Hou, L.-T.; Lai, J.-Y.; Wang, D.-M. Preparation of Porous Scaffolds by Using Freeze-Extraction and Freeze-Gelation Methods. Biomaterials 2004, $25,129-138$.

(6) Salerno, A.; Fernández-Gutiérrez, M.; del Barrio, J. S. R.; Pascual, C. D. Macroporous and Nanometre Scale Fibrous PLA and PLA-HA Composite Scaffolds Fabricated by a Bio Safe Strategy. RSC Adv. 2014, 4, 61491-61502.

(7) Cardea, S.; Baldino, L.; Pisanti, P.; Reverchon, E. 3-D PLLA Scaffolds Formation by a Supercritical Freeze Extraction Assisted Process. J. Mater. Sci.: Mater. Med. 2014, 25, 355-362.

(8) Karim, Z.; Mathew, A. P.; Grahn, M.; Mouzon, J.; Oksman, K. Nanoporous Membranes with Cellulose Nanocrystals as Functional Entity in Chitosan: Removal of Dyes from Water. Carbohydr. Polym. 2014, 112, 668-676.

(9) Hilal, N.; Ismail, A. F.; Wright, C. Membrane Fabrication; CRC Press, 2015.

(10) Matsuyama, H.; Okafuji, H.; Maki, T.; Teramoto, M.; Kubota, N. Preparation of Polyethylene Hollow Fiber Membrane via Thermally Induced Phase Separation. J. Membr. Sci. 2003, 223, 119-126.

(11) Tanaka, T.; Lloyd, D. R. Formation of Poly(L-Lactic Acid) Microfiltration Membranes via Thermally Induced Phase Separation. J. Membr. Sci. 2004, 238, 65-73.

(12) Ho, S. T.; Hutmacher, D. W. A Comparison of Micro CT with Other Techniques Used in the Characterization of Scaffolds. Biomaterials 2006, 27, 1362-1376.

(13) Boller, E.; Tafforeau, P.; Ludwig, W.; Helfen, L.; Weitkamp, T.; Salvo, L.; Cloetens, P.; Baruchel, J. Techniques d'imagerie Pour La Caractérisation 3D Des Matériaux à l'ESRF. In Matériaux 2010; Nantes, France, 2010; p.

(14) Weitkamp, T.; Tafforeau, P.; Boller, E.; Cloetens, P.; Valade, J.P.; Bernard, P.; Peyrin, F.; Ludwig, W.; Helfen, L.; Baruchel, J. Status and Evolution of the ESRF Beamline ID19. In AIP Conference Proceedings; AIP Publishing, 2010; Vol. 1221, pp 33-38.

(15) Buffiere, J.-Y.; Maire, E.; Adrien, J.; Masse, J.-P.; Boller, E. In Situ Experiments with X Ray Tomography: An Attractive Tool for Experimental Mechanics. Exp. Mech. 2010, 50, 289-305.

(16) Salvo, L.; Cloetens, P.; Maire, E.; Zabler, S.; Blandin, J. J.; Buffière, J. Y.; Ludwig, W.; Boller, E.; Bellet, D.; Josserond, C. X-Ray Micro-Tomography an Attractive Characterisation Technique in Materials Science. Nucl. Instrum. Methods Phys. Res., Sect. B 2003, 200, 273-286.

(17) Salerno, A.; Pascual, C. D. Bio-Based Polymers, Supercritical Fluids and Tissue Engineering. Process Biochem. 2015, 50, 826-838.

(18) O'Brien, F. J. Biomaterials \& Scaffolds for Tissue Engineering. Mater. Today 2011, 14, 88-95.

(19) Hutmacher, D. W. Scaffold Design and Fabrication Technologies for Engineering Tissues-State of the Art and Future Perspectives. J. Biomater. Sci., Polym. Ed. 2001, 12, 107-124.
(20) Oliveira, J.; Brichi, G. S.; Marconcini, J. M.; Mattoso, C.; Henrique, L.; Glenn, G. M.; Medeiros, E. S. Effect of Solvent on The Physical and Morphological Properties of Poly(Lactic Acid) Nanofibers Obtained by Solution Blow Spinning. J. Eng. FIBERS Fabr. 2014, 9, 117.

(21) Hua, F. J.; Park, T. G.; Lee, D. S. A Facile Preparation of Highly Interconnected Macroporous Poly(d,l-Lactic Acid-Co-Glycolic Acid) (PLGA) Scaffolds by Liquid-liquid Phase Separation of a PLGAdioxane-water Ternary System. Polymer 2003, 44, 1911-1920.

(22) Schneider, C. A.; Rasband, W. S.; Eliceiri, K. W. NIH Image to ImageJ: 25 Years of Image Analysis. Nat. Methods 2012, 9, 671-675.

(23) Doube, M.; Kłosowski, M. M.; Arganda-Carreras, I.; Cordelières, F. P.; Dougherty, R. P.; Jackson, J. S.; Schmid, B.; Hutchinson, J. R.; Shefelbine, S. J. BoneJ: Free and Extensible Bone Image Analysis in Image J. Bone 2010, 47, 1076-1079.

(24) Hildebrand, T.; Rüegsegger, P. A New Method for the Modelindependent Assessment of Thickness in Three-dimensional Images. J. Microsc. 1997, 185, 67-75.

(25) Dougherty, R.; Kunzelmann, K.-H. Computing Local Thickness of 3D Structures with ImageJ. Microsc. Microanal. 2007, 13, 16781679.

(26) Gay, S.; Lefebvre, G.; Bonnin, M.; Nottelet, B.; Boury, F.; Gibaud, A.; Calvignac, B. PLA Scaffolds Production from Thermally Induced Phase Separation: Effect of Process Parameters and Development of an Environmentally Improved Route Assisted by Supercritical Carbon Dioxide. J. Supercrit. Fluids 2018, 136, 123-135. 